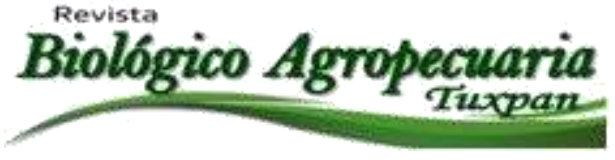

\title{
Proyecto de inversión: modernización de la granja lechera de la facultad de ciencias agrícolas y forestales en Cd. Delicias, Chihuahua
}

Investment project: modernization of the dairy farm of the Faculty of Agricultural and Forestry Sciences in Ciudad Delicias, Chihuahua

\author{
Magaña-Magaña José Eduardo ${ }^{\bowtie}$, Magda Ivett Mejía-Saavedra ${ }^{2}$, Víctor Hugo Villarreal-Ramirez ${ }^{3}$, \\ Lucero Beatriz-Rivas ${ }^{4}$ y Javier Núñez-Lopez ${ }^{6}$
}

Agronegocios de la Facultad de Ciencias Agrícolas y Forestales de la Universidad Autónoma de Chihuahua $^{13,4,6}$ profesores de la Facultad de Ciencias Agrícolas y Forestales de la Universidad Autónoma de Chihuahua.

Autor para correspondencia: emagana@uach.mx

Recibido: 07/01/2014

Aceptado: 19/07/2014

\section{RESUMEN}

El propósito del estudio fue contribuir a la modernización del establo de la Facultad de Ciencias agrícolas y Forestales de la Universidad Autónoma de Chihuahua, para ser un ejemplo de prototipo tecnológico para los pequeños productores de leche de la región con hatos de 40 a 60 vacas. El análisis interno mostró que el área de producción no cuenta con registros individuales que le permita llevar un adecuado control de egresos e ingresos; en el área de nutrición se carece de dietas adecuadas para cada tipo de animal (becerras, vaquillas, vacas en producción). En lo referente a la infraestructura, las instalaciones no cuentan con el mantenimiento requerido y el equipo es obsoleto. El establo actualmente tiene 38 vacas. Los forrajes que consume son producidos en el área agrícola de la misma escuela, considerando precios de mercado. Se proyectó una ampliación del hato a 50 vacas lecheras y se formuló un proyecto de inversión, cumpliendo los objetivos planteados. Una vez analizados los egresos e ingresos y calculado el flujo neto de efectivo, el proyecto nos arroja un resultado de una TIR de $42 \%$ y un VAN al $15 \%$ de $\$ 1,328,888.00$, lo que financieramente nos indica que el proyecto es viable. Se condujo un análisis de sensibilidad con los escenarios de incremento de costos de un $10 \%$, disminución de precios de un $10 \%$ y un escenario final de incremento de costos y disminución de precios, teniendo como resultados una TIR de $24 \%$ y un VAN de $\$ 416,489.09$, lo que demuestra la robustez financiera del proyecto.

Palabras claves: Modernización, Inversión, Establo, Evaluación Económica, Análisis de Sensibilidad. 


\section{ABSTRACT}

The present research was made in the Facultad de Ciencias Agrícolas y Forestales, UACH during the period 2011-2013. The general objective of the research was to contribute the modernization of the cowshed of FCA y F in order to make it more productive. The results of the study showed that the production area does not count with individual records which could allow taking control of costs and expenses in the area of nutrition. There are no adequate diets for each different animal (yearlings, calves and cattle in production). In relation to the infrastructure, the equipment does not count with the required maintenance and therefore it is obsolete. The cowshed has some favorable aspects such as basic subsistent labor services (water, electricity, etc.) and the forage which is given to cows are products of the same school. With the information obtained a Project to increase herd was designed to have fifty milking cows and a Project of inversion was also formulated achieving the objectives. The intention of the Project is for the motivation of the present administration has to modernize and therefore achieving the objective planned. Once the analyzed of cost and incomes are completed calculating the fluency of cash the projects shows a result of a TIR of $42 \%$ and a VAN of $\$ 1,328,888$ which financially indicates that the Project is viable. An analysis of sensibility of state of costs of a $10 \%$ and a down in prices, having as a result a TIR of $24 \%$ and a VAN of $\$ 416,689.09$ which shows the financial robust of the Project.

Keywords: Modernization, Investment, Economical Evaluation, Analysis of sensibility.

\section{INTRODUCCIÓN}

El mercado internacional de lácteos es volátil e inestable y su tendencia general es mantener e incluso incrementar los precios, una vez se atenúe la crisis financiera internacional. Por ello, los países importadores deben enfocar el desarrollo lechero hacia el incremento de la producción nacional hasta cubrir toda la demanda interna, combinando la sostenibilidad de los sistemas intensivos con el desarrollo local de los pequeños y medianos productores. Considerando que los precios de los insumos se mantendrán relativamente altos en relación a los precios de la leche, México tiene una clara desventaja competitiva al competir hombre animal por los alimentos como el maíz que es parte de la dieta esencial del mexicano y otros alimentos.

Se requiere de un nuevo enfoque de desarrollo donde la organización y administración de la finca lechera, la aplicación de la ciencia, la técnica y la integración en cadenas tienen un papel fundamental. Aunque el sector lechero mexicano ha estado afectado por múltiples limitaciones en los últimos 15 años y depende en cerca del 70 por ciento de las importaciones, existen las condiciones socio-políticas, infraestructurales y de preparación

técnica para lograr una profunda transformación en el futuro mediato.

Durante las últimas décadas la producción de leche, en muchas partes del mundo, ha sufrido una gran revolución;

\section{Revista Científica Biológico Agropecuaria Tuxpan 2 (1)}


revolución que todavía está en progreso. La leche se produce por menor número de ganado vacuno, pero con una mayor producción individual. Los cambios estructurales han causado una disminución de las granjas de producción lechera, pero han incrementado, éstas, su tamaño y tecnología. Esta última ha sido la herramienta clave para el manejo del establo.

La ordeña del ganado vacuno es la parte central del manejo del establo lechero para optimizar la capacidad de producción y la calidad de leche. El ordeño no es sólo un proceso donde la leche se extrae de los pezones; es mucho más, es un proceso en el que muchos mecanismos fisiológicos son activados en el organismo de la vaca lechera, regulando la capacidad de producción, la composición de la leche, el consumo de alimento y el propio comportamiento del animal (Dairy Values, 2005).

\section{REVISIÓN DE LITERATURA}

Reaves y Pegram (1974), dicen que la vaca lechera en producción proporciona diariamente al ganadero un producto comercial de venta inmediata. Produce por día mayor cantidad de alimento para el hombre que cualquier otro animal. La leche que produce constituye una fuente regular de ingresos, mucho mayor que el que se obtiene de cualquier otro animal doméstico.

McMeekan (1960) menciona que la eficiente producción de leche está determinada por cierto número de factores básicos interactuantes de los cuales los más importantes son el rendimiento y el tamaño de la vaca, y su nivel de alimentación. Los factores de manejo modifican en grado considerable el comportamiento. El estado de la vaca en la parición, longitud del periodo seco, la fecha de parición, la duración del periodo del servicio y de la lactancia, el manejo del ordeño y la incidencia de enfermedades, todo debe ser mantenido bajo constante vigilancia para un eficiente producción del hato lechero.

Shimada (1983), nos indica que la nutrición es la rama más importante de la producción animal, tanto desde el punto de vista cuantitativo como económico, ya que si se analizan los costos de producción pecuaria subrayan el papel sobresaliente de esta. En el caso de los rumiantes, el costo de producción varía según sean los animales explotados en el corral. Las vacas lecheras estabuladas tienen un costo de aproximadamente el $70 \%$ por concepto de alimentación.

Bonhomme, D. (1970), dice que la producción bovina supone la utilización de una serie de técnicas que, aplicadas a un ente biológico, el animal transformador, buscan la remuneración bajo forma de carne, leche y estiércol, partiendo de capitales de diferentes orígenes.

\section{METODOLOGÍA}

\section{Formato proyecto FIRA}

Para el diseño del proyecto se utilizo el formato FIRA (Fideicomisos Instituidos en Relación con la Agricultura). 


\section{RESULTADOS}

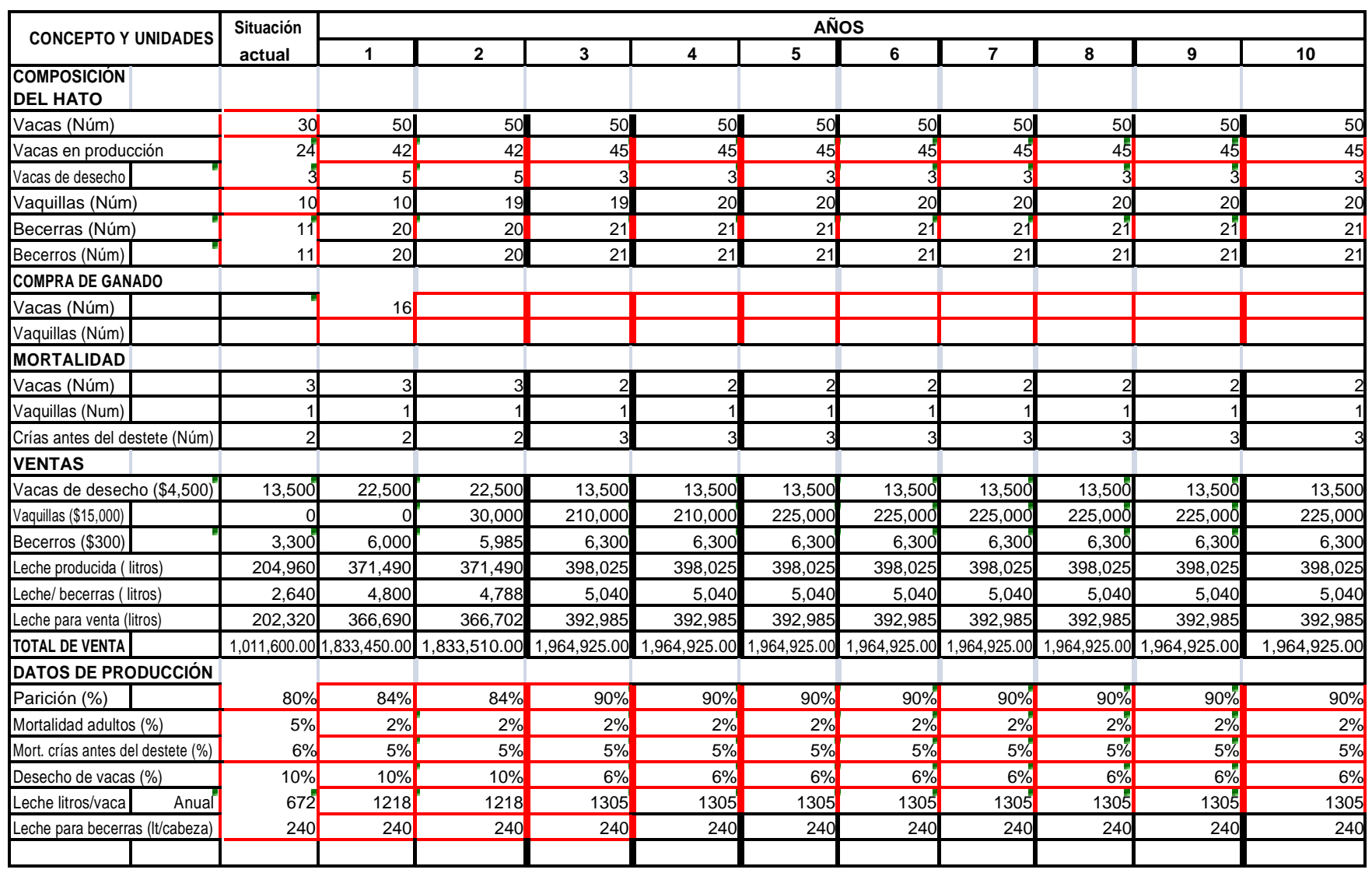

\section{Situación financiera (actual y proyectada)}

Ampliación del establo a 50 vacas. 
Magaña et al., 2014

\begin{tabular}{|c|c|c|c|c|c|c|c|c|}
\hline CONCEPTO & AÑO 0 & AÑO 1 & AÑO 2 & AÑO 3 & AÑO 4 & AÑO 5 & AÑO 6 & AÑO 7 \\
\hline \multicolumn{9}{|l|}{ Ingresos } \\
\hline leche & & $\$ 1,833,450$ & $\$ 1,833,510$ & $\$ 1,964,925$ & $\$ 1,964,925$ & $\$ 1,964,925$ & $\$ 1,964,925$ & $\$ 1,964,925$ \\
\hline vacas desecho & & $22,500.00$ & $22,500.00$ & $13,500.00$ & $13,500.00$ & $13,500.00$ & $13,500.00$ & $13,500.00$ \\
\hline vaquillas & & 0 & 30,000 & 210,000 & 210,000 & 225,000 & 225,000 & 225,000 \\
\hline becerros & & 6,000 & 5,985 & 6,300 & 6,300 & 6,300 & 6,300 & 6,300 \\
\hline Sub-total & & $\$ 1,861,950$ & $\$ 1,891,995$ & $\$ 2,194,725$ & $\$ 2,194,725$ & $\$ 2,209,725$ & $\$ 2,209,725$ & $\$ 2,209,725$ \\
\hline & & & & & & & & \\
\hline $\begin{array}{l}\text { Egresos } \\
\text { alimentacion vacas produccion }\end{array}$ & & $848,842.45$ & $848,842.45$ & $848,842.45$ & $848,842.45$ & $848,842.45$ & $848,842.45$ & 848.842 .45 \\
\hline alimentacion vacas secas & & $10,853.14$ & $10,853.14$ & $10,853.14$ & $10,853.14$ & $10,853.14$ & $10,853.14$ & $10,853.14$ \\
\hline alimentacion vaquillas & & $156,315.30$ & $156,315.30$ & $156,315.30$ & $156,315.30$ & $156,315.30$ & $156,315.30$ & $156,315.30$ \\
\hline alimentacion becerras & & $9,216.60$ & $9,216.60$ & $9,216.60$ & $9,216.60$ & $9,216.60$ & $9,216.60$ & $9,216.60$ \\
\hline agua & & $16,800.00$ & $16,800.00$ & $16,800.00$ & $16,800.00$ & $16,800.00$ & $16,800.00$ & $16,800.00$ \\
\hline energia electrica & & $48,000.00$ & $48,000.00$ & $48,000.00$ & $48,000.00$ & $48,000.00$ & $48,000.00$ & $48,000.00$ \\
\hline mano de obra & & $\$ 148,920$ & $148,920.00$ & $148,920.00$ & $148,920.00$ & $148,920.00$ & $148,920.00$ & $148,920.00$ \\
\hline Sub-total & & $1,238,947.48$ & $\$ 1,238,947.48$ & $1,238,947.48$ & $1,238,947.48$ & $\$ 1,238,947.48$ & $1,238,947.48$ & $1,238,947.48$ \\
\hline Utilidad Bruta & & $\$ 623,002.52$ & $\$ 653,047.52$ & $\$ 955,777.52$ & $\$ 955,777.52$ & $\$ 970,777.52$ & $\$ 970,777.52$ & $\$ 970,777.52$ \\
\hline Depreciacion & & 300,863 & 300,863 & 300,863 & 300,863 & 300,863 & 300,863 & 300,863 \\
\hline Utilidad despues de depreciacion & & $\$ 322,139.92$ & $\$ 352,184.92$ & $\$ 654,914.92$ & $\$ 654,914.92$ & $\$ 669,914.92$ & $\$ 669,914.92$ & $\$ 669,914.92$ \\
\hline Impuestos & & $\$ 64,427.98$ & $\$ 70,436.98$ & $\$ 130,982.98$ & $\$ 130,982.98$ & $\$ 133,982.98$ & $\$ 133,982.98$ & $\$ 133,982.98$ \\
\hline & & & & & & & & \\
\hline Utilidad despues de impuestos & & $\$ 257,711.93$ & $\$ 281,747.93$ & $\$ 523,931.93$ & $\$ 523,931.93$ & $\$ 535,931.93$ & $\$ 535,931.93$ & $\$ 535,931.93$ \\
\hline Depreciacion & & 300,863 & 300,863 & 300,863 & 300,863 & 300,863 & 300,863 & 300,863 \\
\hline & & & & & & & & \\
\hline Flujo de efectivo & $-1,504,313$ & $\$ 558,574.53$ & $\$ 582,610.53$ & $\$ 824,794.53$ & $\$ 824,794.53$ & $\$ 836,794.53$ & $\$ 836,794.53$ & $\$ 836,794.53$ \\
\hline & & & & & & & & \\
\hline TIR & $42 \%$ & & & & & & & \\
\hline $\operatorname{VAN}(15 \%)$ & $\$ 1,328,888.87$ & & & & & & & \\
\hline
\end{tabular}

Análisis de rentabilidad (a precios y valores constantes)

TIR

$\operatorname{VAN}(15 \%)$
$42 \%$

$\$ 1,328,888.87$

\section{Análisis de sensibilidad}

En el análisis de sensibilidad cuando se disminuye un $10 \%$ del precio de la leche se observa una disminución de la TIR de un $42 \%$ a un $31 \%$ y del VAN de $\$ 1,328,888.87$ a $775,065.01$.

Cuando los costos aumentan a un $10 \%$ la disminución de la TIR y VAN es menor que cuando los precios disminuyen. TIR $35 \%$ y VAN \$970,312.07.

Estos dos indicadores financieros manifiestan una disminución más significativa cuando se utiliza la combinación de un decremento de precios y un aumento de costos a un $10 \%$. TIR $24 \%$ y VAN \$416,489.09.

Estamos utilizando un TREMA (Taza de Rentabilidad Mínima Aceptada) igual a 15\% y analizando la ultima combinación podemos concluir que todavía es rentable, lo que nos indica una robustez del proyecto.

\section{CONCLUSIONES}

De acuerdo a los resultados obtenidos en el presente estudio, se puede concluir que la unidad de producción presenta diversas

\section{Revista Científica Biológico Agropecuaria Tuxpan 2 (1) ISSN: 2007-6940}


deficiencias tanto técnicas como económicas, lo que no ha permitido que se desarrolle favorablemente y apoye a la formación de los alumnos. Además el equipo con el que cuenta está obsoleto.

Se pretende adquirir equipos modernos de ordeña y mejorar las construcciones por la cantidad de $\$ 1,504,313$ para mejorar la calidad genética de los animales ampliando el hato a 50 vacas. Una vez analizados los costos y los ingresos y calculado el flujo neto de efectivo, el proyecto nos arroja un resultado de un TIR de $42 \%$ y un VAN de $\$ 1,328,888$, lo que financieramente nos indica que el proyecto es viable.

Además se realizaron tres análisis de sensibilidad, en el primero se hiso un decremento en los ingresos y obtuvimos un TIR de $31 \%$ y un VAN de $\$ 775,065.01$ (ver anexo E), en el segundo análisis aumentamos los costos y obtuvimos un TIR de $35 \%$ y un VAN de $\$ 970,312.07$ (ver anexo F), por ultimo en el tercer análisis conjuntamente se aumentaron los costos y decrementamos los ingresos y como resultado obtuvimos un TIR de $24 \%$ y un VAN de $\$ 416,489.09$ (ver anexo D), lo que nos indica una robustez en el proyecto.
Se recomienda que el proceso de modernización se lleve a cabo mediante la dirección del responsable del establo el Maestro Manuel Lujan Favela.

\section{LITERATURA CITADA}

Bonhomme, D. 1970. Explotación del ganado vacuno. Ediciones Mundi Prensa. Madrid España.

Centro de estadística agropecuaria SAGAR. Boletín bimestral de leche.

Shimada, A.S. 1983. Fundamentos de nutrición animal comparativa. México.

Magaña, M. E. e Licon, V. I. 2010. Estudio de Caso "Formulación y Evaluación de un Proyecto para instalar 3ha de invernadero para producir fresa". Facultad de Ciencias Agrícolas y Forestales. México.

Reaves, P. M y Pegram, C. W. 1974. El ganado lechero y la industria láctea en la granja. Editorial Limusa. México.

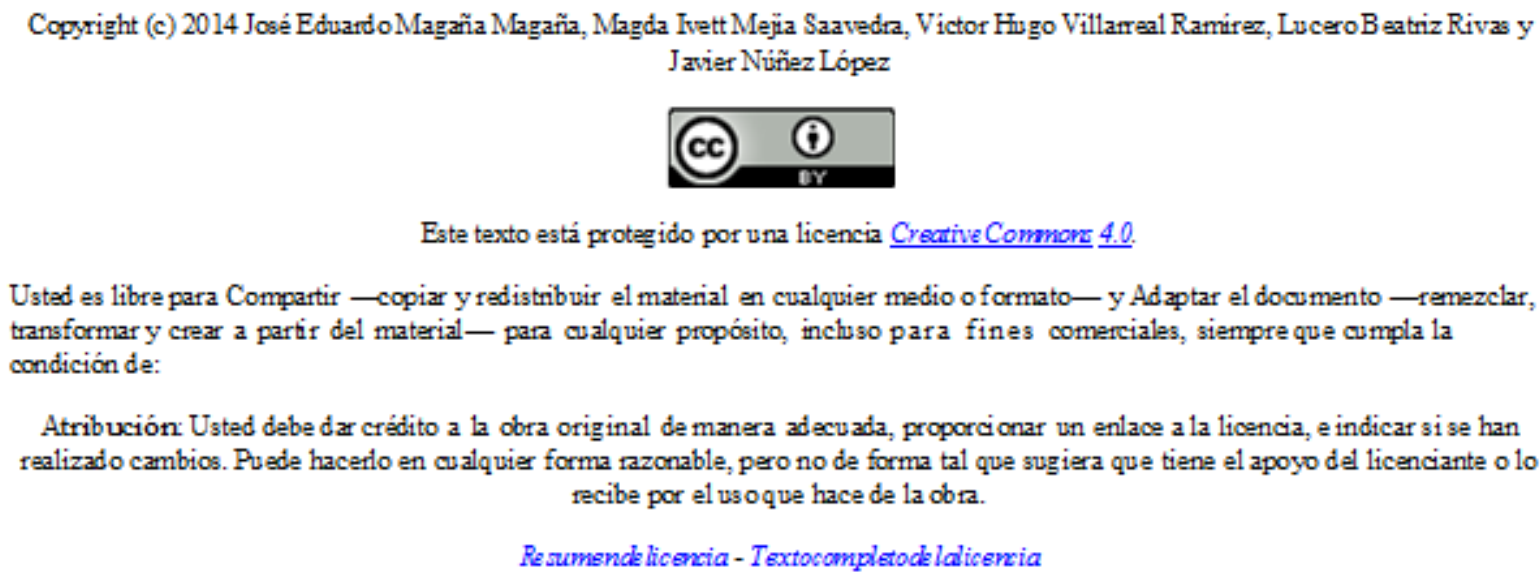

